Bio - grafia. Escritos sobre la Biología y su Enseñanza. ISSN 2027

Edición Extraordinaria. p.p. 1348-1359

Memorias del VIII Encuentro Nacional de Experiencias en Enseñanza de la Biología y la Educación Ambiental. III Congreso Nacional de Investigación en Enseñanza de la Biología.

\title{
EL CONCEPTO DE EVOLUCIÓN: UN NUEVO CONOCIMIENTO MÁS AMPLIO Y AJUSTADO A LA REALIDAD CIENTÍFICA EN ESTUDIANTES DE NOVENO GRADO A PARTIR DE UNA RECONCEPTUALIZACIÓN
}

\author{
1. E. Parra Pérez \\ 2. I. Ortiz Arévalo \\ 3. S. Reyes Castro
}

\section{RESUMEN}

El estudiante, en cuanto indaga permanentemente por el saber, ambicionando establecer un equilibrio con sus discernimientos, busca respuestas, se plantea interrogantes, investiga y descubre, hasta llegar al conocimiento que le hace volver de nuevo al equilibrio cognitivo, de tal manera que la resolución de este conflicto se obtiene como resultado el aprendizaje, logrando que se reconfigure el esquema cognitivo previo. De acuerdo con lo anterior, el presente artículo da cuenta de la experiencia pedagógica realizada en el I.E.D Colegio San Martín de Porres ubicado en Bogotá, localidad de Chapinero, con estudiantes de noveno grado de la jornada de la tarde con edades entre los 14 y 19 años, bajo el enfoque de conflicto cognitivo. Con el objetivo de romper la estructura cognitiva del alumno, generando una nueva reequilibración, resultado de un conocimiento enriquecido, se implementó la unidad didáctica: "INTERPRETACIÓN DE LA EVOLUCIÓN: Enseñanza desde el conflicto cognitivo" guiando este concepto por tres condiciones: desafío, equilibro entre lo fácil y lo difícil, y su entendimiento. Evidenciando en los resultados obtenidos, un cambio conceptual que tuvo cada estudiante con respecto al concepto de evolución y los temas que sirvieron de mecanismo de abordaje. Concluyendo así que es posible despertar el interés de los estudiantes, al desarrollar el componente afectivo y de interrelación con el diario vivir, los condujo al fortalecimiento y manejo del aprendizaje científico.

PALABRAS CLAVE: Conflicto cognitivo, evolución, aprendizaje, conocimiento previo, concepciones alternativas.

\section{ABSTRACT:}

The student, upon permanently investigates for knowledge, coveting a balance with their insights, seeks answers, questions arise, investigate and discover, to come to the 
Bio - grafia. Escritos sobre la Biología y su Enseñanza. ISSN 2027

Edición Extraordinaria. p.p. 1348-1359

Memorias del VIII Encuentro Nacional de Experiencias en Enseñanza de la Biología y la Educación Ambiental. III Congreso Nacional de Investigación en Enseñanza de la Biología.

knowledge that brings him back again to cognitive balance, so that the resolution This conflict is obtained as a result learning, making cognitive reconfigure previous scheme. According to the above, this article reports on the educational experience at the IED Colegio San Martin de Porres located in Bogota, Chapinero locality, with freshmen of the day in the afternoon between the ages of 14 and 19 years, under the approach of cognitive conflict. In order to break the student's cognitive structure, creating a new rebalancing, resulting from an enriched knowledge, teaching unit was implemented: "INTERPRETATION OF EVOLUTION: Teaching from the cognitive conflict" guiding concept for three conditions: challenge, balance between the easy and the difficult, and understanding. Showing in the results, a conceptual change that took each student to the concept of evolution and the issues that served mechanism approach. Thus concluding that it is possible to interest students in developing affective component and interaction with daily life, he led to the strengthening and management of scientific learning.

KEYWORDS: Cognitive Conflict, development, learning, prior knowledge, alternative conceptions.

\section{INTRODUCCIÓN}

Desde que un individuo nace entra hacer encasillado de acuerdo a diversos patrones, ya sea el tono de su piel, la escala social de los padres, entre otros, es decir las características que median su origen, logrando categorizarlo en el grupo al cual corresponde, dada las similitudes presentes con dicho grupo de manera más cercana. Pero qué hay más allá de esa primera respuesta establecida en torno a una parte de la categorización de los seres vivos presentes en el planeta tierra, cuyo fundamento aplica una metodología de lo más amplio a lo más pequeño, instaurando un parentesco según los conocimientos instaurados en la ciencia. A simple vista esta forma de organizar a los seres presentes en la naturaleza deja en el aire muchas dudas respecto al parentesco y diferencias enmarcadas con los otros grupos, planteando varias preguntas en los alumnos ¿Cuál es el origen de la vida? ¿Cómo se estableció los diferentes grupos de seres vivos en él planeta? ¿Qué pasa con el origen de nuestra especie? ¿Quién fue nuestro ancestro, que hizo posible nuestra existencia? Colocando al alumno en la búsqueda de respuestas, que permitan comprender el origen del universo y lo que ello abarca. De dicho proceso se posibilita la constitución y el avance de su pensamiento, dando respuesta de manera más cercana a los hechos con base a los 
Bio - grafia. Escritos sobre la Biología y su Enseñanza. ISSN 2027

Edición Extraordinaria. p.p. 1348-1359

Memorias del VIII Encuentro Nacional de Experiencias en Enseñanza de la Biología y la Educación Ambiental. III Congreso Nacional de Investigación en Enseñanza de la Biología.

conocimientos científicos, producto de varias contribuciones de diferentes pensadores como Darwin, Albert Einstein, Ronald Fisher, Sewall Green Wright, Ernst Mayr, entre muchos otros.

Es entonces que el aprendizaje de las ciencias (Física, Química, Biología, Matemática, etc.), se ha convertido en una necesidad educativa con el fin de compartir los avances que en sus diferentes campos se han logrado establecer hasta el momento, permitiendo un acercamiento a la verdad e influenciando un poco el reflejo de lo que podría ser el futuro. Recayendo como obligación en la humanidad el ser partícipe de los conocimientos instaurados y transmitirlos conforme a la cultura, para ello la educación se establece como un derecho permitiendo la formación de cada individuo. Y contribuyendo con ello, se implementó la Unidad Didáctica que permitiera llevar a cabo la enseñanza y aprendizaje de conceptos sobre evolución, como una forma de facilitar la comprensión y construcción del conocimiento en los estudiantes a través de ideas previas y diferentes perspectivas con el apoyo del diseño de estrategias y metodologías que permitan avanzar en diferentes temas en el área de la Biología.

La educación, en el marco político a nivel de la instituciones educativas en el territorio nacional, se orienta como un proceso de formación permanente, personal, cultural y social que se fundamenta en una concepción integral de la persona humana, de su dignidad, sus derechos y de sus deberes, trasbordando al estudiante a experimentar en todo el proceso de enseñanza y aprendizaje en la escuela un reconocimiento de su ser configurado con apreciaciones del mundo a través de los conceptos manejados en las diferentes áreas de la ciencia, llegándose a enfrentar con múltiples paradigmas que contradicen sus esquemas previos y exigen el establecimiento de una fase de asimilación, ocasionando conflicto. Dicho conflicto entre conocimientos en los estudiantes es posible abordarlo a partir del enfoque de "conflicto cognitivo" siendo útil en diferentes áreas educativas, por ejemplo en la enseñanza de la biología, donde el educador crea conflicto en el estudiante mediante la presentación de una situación problema, de manera que éstos pongan a prueba sus concepciones alternativas para lograr un cambio conceptual, se trata de partir de las concepciones alternativas de los alumnos confrontándolas con situaciones conflictivas, para lograr un cambio conceptual, entendido como su sustitución por otras teorías más potentes, es decir más próximas al conocimiento científico. Aunque debe ser el propio 
Bio - grafia. Escritos sobre la Biología y su Enseñanza. ISSN 2027

Edición Extraordinaria. p.p. 1348-1359

Memorias del VIII Encuentro Nacional de Experiencias en Enseñanza de la Biología y la Educación Ambiental. III Congreso Nacional de Investigación en Enseñanza de la Biología.

alumno el que tome conciencia de ese conflicto y lo resuelva, los educadores pueden utilizar todos los recursos, expositivos y no expositivos, a su alcance para hacer ver al alumno las insuficiencias de sus propias concepciones (Pozo, 1997)

Según Pozo (1997), el rol del estudiante es construir su propio conocimiento, tomar conciencia de la situación problema planteada por el educador, proponer de manera activa soluciones, investigar, evaluar sus soluciones y adecuarse frente a las nuevas posibilidades del modelo científico; el rol del profesor es proponer una situación problema, mediar y acompañar en el aprendizaje desde el conocimiento cotidiano hacia el conocimiento científico y evaluar el aprendizaje hacia el logro de habilidades cognitivas de orden superior, basado en núcleos conceptuales, contenidos organizados jerárquicamente y actividades secuenciadas para que el estudiante ponga a prueba su propio sistema de referencias con el fin de que la nueva concepción sea convincente y creíble para el estudiante. Partiendo de lo anterior la meta de la educación científica es concientizar al estudiante que es más provechoso solucionar problemas cognitivos a través de un modelo científico con más potencia teórica.

\section{METODOLOGÍA}

La Unidad Didáctica se desarrolló en el colegio San Martin de Porres IED, ubicado en la Calle 45 \# 3 - 35 Este, barrió San Martín perteneciente a la localidad de Chapinero, estando dirigido a 30 estudiantes del grado 901. Con quienes se llevó a cabo la comprensión del concepto de evolución mediante el enfoque Conflicto Cognitivo el cual señala como eje principal el desarrollo del pensamiento científico de los estudiantes a partir del contraste de sus ideas previas frente al conocimiento científico generando una construcción y apropiación del conocimiento que implica, así como lo señala Pozo (2004), un cambio profundo a nivel conceptual, actitudinal y metodológico.

La propuesta para la implementación de la unidad didáctica partió del conocimiento cotidiano e ideas previas de los alumnos, sin considerarlas erróneas, buscando incluirlas en las actividades propuestas al desarrollar cada clase. Además del contraste con los conocimientos establecidos a nivel científico en la búsqueda de respuestas a los paradigmas que giran en torno a la evolución. Para ello, al comienzo de cada clase se indago por medio de una red conceptual las asimilaciones y comprensiones que tienen los 
Bio - grafia. Escritos sobre la Biología y su Enseñanza. ISSN 2027

Edición Extraordinaria. p.p. 1348-1359

Memorias del VIII Encuentro Nacional de Experiencias en Enseñanza de la Biología y la Educación Ambiental. III Congreso Nacional de Investigación en Enseñanza de la Biología.

estudiantes referentes al tema a tratar en la clase, para luego realizar un contraste con las opiniones del grupo y llegar a un punto intermedio, pasando en seguida a analizar los preceptos científicos valiéndose de las Tics; ayudas lúdicas como lo son videos, presentaciones, etc.

La transformación y apropiación del conocimiento del estudiante, intenta determinarse por el cuestionamiento y aclaraciones de las dudas producto del estudiante, pretendiendo no caer en el error de imponer los conceptos de la ciencia ni mucho menos los del docente. Además, se implementó la aplicación de actividades que buscaran captar el interés por parte de los estudiantes en el tema y se dieran las posibilidades para la transformación del conocimiento.

Las herramientas utilizadas para la realización de la unidad didáctica en primer lugar fueron la caracterización social de los estudiantes, del profesor titular y de la institución educativa en general, con el fin de entender la población y obtener información que permitirá el pleno desarrollo de la unidad didáctica, cuya información se obtuvo a partir de la implementación de encuestas que a su vez permitieron conocer las ideas previas de los estudiantes acerca del concepto de evolución, para qué de esta manera se pudieran planear estratégicamente cada una de las clases desde el concepto de fósil, eras geológicas, Big Bang, gran extinción de los dinosaurios hasta los caracteres evolutivos humanos, ideas previas que sirvieron para la reestructuración de conceptos a través de talleres, videos, problemas y demás que permitieron el transcurso y culminación de la unidad didáctica.

Las actividades realizadas se diseñaron con el fin de que el estudiante tome conciencia de la situación problema que se le plantea, inquiriendo en que el alumno proponga sus propias soluciones por medio de la investigación y el descubrimiento, de tal manera que él se adapte a las nuevas posibilidades del modelo científico. Dichas actividades se plantearon de la siguiente manera como da cuenta en cronograma de actividades en la tabla 1:

Tabla 1

Cronograma de Actividades

\begin{tabular}{l|l|l} 
CLASE & ACTIVIDAD & DESARROLLO
\end{tabular}


Bio - grafia. Escritos sobre la Biología y su Enseñanza. ISSN 2027

Edición Extraordinaria. p.p. 1348-1359

Memorias del VIII Encuentro Nacional de Experiencias en Enseñanza de la Biología y la Educación Ambiental. III Congreso Nacional de Investigación en Enseñanza de la Biología.

\begin{tabular}{|c|c|c|}
\hline 0 & Encuestas & $\begin{array}{l}\text { Se diseñan encuestas con el fin de conocer el contexto } \\
\text { social del estudiante, del aula de clase y del colegio. }\end{array}$ \\
\hline 1 & $\begin{array}{l}\text { ¿Qué son los fósiles y } \\
\text { cómo fue su } \\
\text { formación? }\end{array}$ & $\begin{array}{l}\text { A partir de las ideas previas del estudiante se } \\
\text { transformay se construye un nuevo concepto de fósil. }\end{array}$ \\
\hline 2 & $\begin{array}{l}\text { ¿Qué son las eras } \\
\text { geológicas? }\end{array}$ & $\begin{array}{l}\text { A partir del nuevo concepto de fósil se explican las } \\
\text { diferentes eras geológicas; se culmina con la } \\
\text { construcción de un fósil en greda. }\end{array}$ \\
\hline 3 & $\begin{array}{l}\text { ¿Cómo se dieron los } \\
\text { cambios evolutivos en } \\
\text { los seres vivos? } \\
\text { ¿Siempre fuimos los } \\
\text { mismos? }\end{array}$ & $\begin{array}{l}\text { A partir de interrogantes y problemas se cuestiona al } \\
\text { estudiante de tal manera que se pregunte acerca de la } \\
\text { evolución de los seres vivos ¿Nuestro planeta siempre } \\
\text { fue el mismo? ¿Por qué en Villa de Leyva se encuentran } \\
\text { fósiles marinos?, con el fin de que el estudiante } \\
\text { investigue y encuentre un equilibrio cognitivo. }\end{array}$ \\
\hline 4 & $\begin{array}{l}\text { ¿La Gran Extinción de } \\
\text { los } \quad \text { Dinosaurios } \\
\text { ocurrió a causa del } \\
\text { Big Bang? }\end{array}$ & $\begin{array}{l}\text { Se ubican en una línea del tiempo la teoría del Big Bang } \\
\text { y la teoría de la Gran Extinción de los Dinosaurios con } \\
\text { el fin de dimensionar los tiempos en que ocurrieron } \\
\text { cada uno de los hechos (calendario cósmico), sus } \\
\text { principales características e implicaciones hoy en día. }\end{array}$ \\
\hline 5 & $\begin{array}{l}\text { ¿Qué son los } \\
\text { caracteres evolutivos } \\
\text { humanos? }\end{array}$ & $\begin{array}{l}\text { Reconocimiento del dimorfismo sexual como parte de la } \\
\text { evolución humana. }\end{array}$ \\
\hline 6 & $\begin{array}{l}\text { ¿Cómo se identifican } \\
\text { los cráneos en los } \\
\text { humanos? }\end{array}$ & $\begin{array}{l}\text { Se cuestiona al estudiante acerca de la edad y el sexo } \\
\text { de los cráneos según con el conocimiento construido el } \\
\text { estudiante indaga, investiga y descubre. }\end{array}$ \\
\hline 7 & Evaluación final & $\begin{array}{l}\text { Se evalúan los temas vistos con el fin de observar un } \\
\text { cambio conceptual en el estudiante. }\end{array}$ \\
\hline
\end{tabular}

RESULTADOS Y DISCUSIÓN:

En la primera sesión donde se partió de las ideas previas de los estudiantes, plasmando sus inquietudes y repuestas en un taller, a partir de preguntas problemas tales como: ¿Sabías que existen los fósiles vivientes?, ¿El registro fósil de un dinosaurio es una evidencia de la evolución humana? ¿Podrías fosilizarte? ¿Qué necesitas para ser un fósil?, a modo que se 
Edición Extraordinaria. p.p. 1348-1359

Memorias del VIII Encuentro Nacional de Experiencias en Enseñanza de la Biología y la Educación Ambiental. III Congreso Nacional de Investigación en Enseñanza de la Biología.

posibilitará la construcción de un nuevo concepto a partir del desequilibrio de las estructuras mentales que se producen cuando se platean este tipo de preguntas al estudiante, provocando la necesidad de hacer algo ante la situación que le produjo tal desequilibrio, la primera reacción de los estudiantes fue negar y dejar al lado el problema, sin embargo al intentar interiorizar el concepto el estudiante empezó a interrogarse y cambiar de cierto modo el concepto de fósil, cuestionando su importancia dentro de la ciencia y la cultural. Como actividad final de la sesión los estudiantes expresaron la determinación a la cual pudieron llegar sobre el concepto, obteniendo como respuesta una postura adaptativa en donde el estudiante toma conciencia de la perturbación e intenta resolver dicho cuestionamiento.

En la segunda sesión obtenido el nuevo concepto de fósil se explicaron las diferentes eras geológicas y como es posible tener un registro de ellas a partir de los procesos de fosilización. Se culminó la clase realizando un fósil en greda, como lo evidencia las fotos de la las figuras 1, 2, 3 y 4, con el fin de acercar al estudiante al concepto a través de la acción, debido a que muchos de los alumnos pensaban que solo los huesos y los dientes de los animales eran los únicos que podían fosilizarse y no consideraban que las huellas, las perforaciones, los organismos mismos o partes desmembradas y aisladas del cuerpo $e$
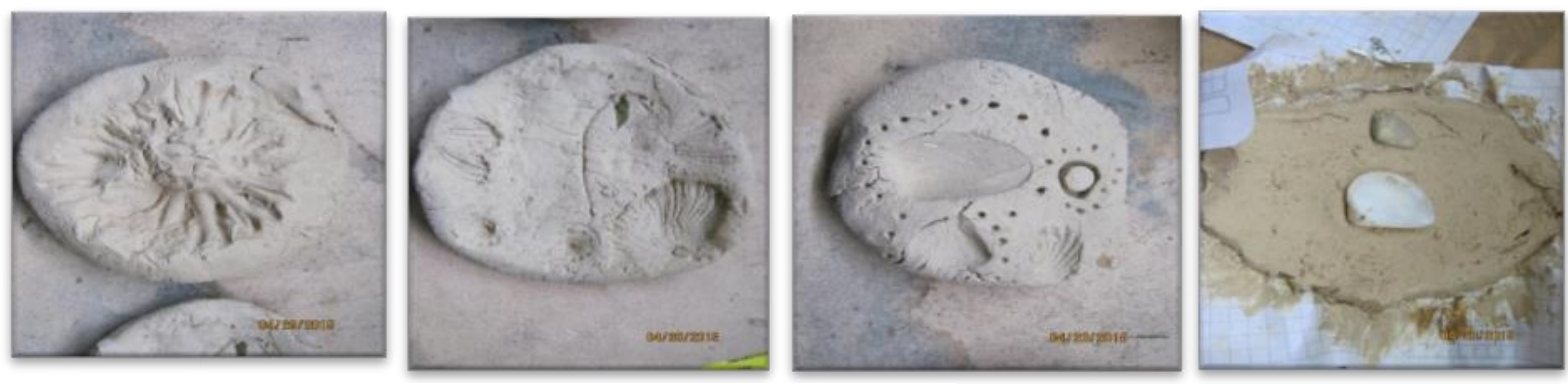

Figura 1, 2, 3 y 4 Construcción de fósil en greda

son denominados fósiles.

En la tercera sesión denominada ¿Cómo se dieron los cambios evolutivos? ¿Siempre fuimos los mismos?, se planeó con el fin de cuestionar al estudiante acerca de la evolución de los humanos partiendo de preguntas tales como: clos humanos vienen de los simios, entonces por que aún hay simios sin evolucionar? ¿Qué nos hizo evolucionar? ¿Por qué se habla de evolución si nadie la ha visto?, ¿Nuestro planeta siempre fue el mismo? ¿Por qué en Villa de 
Bio - grafia. Escritos sobre la Biología y su Enseñanza. ISSN 2027

Edición Extraordinaria. p.p. 1348-1359

Memorias del VIII Encuentro Nacional de Experiencias en Enseñanza de la Biología y la Educación Ambiental. III Congreso Nacional de Investigación en Enseñanza de la Biología.

Leyva se encuentran fósiles marinos?, de tal manera de que el estudiante empezara a cuestionarse acerca de la evolución de su propio cuerpo con respecto al ambiente y el mundo en que convive desde una perspectiva científica.

En la cuarta sesión denominada Teoría del Big Bang versus la Gran Extinción de los Dinosaurios, se pensó con el fin que los estudiantes realizaran la diferenciación de estos dos hechos que plantea la ciencia, dado que confundían las dos teorías como una sola. Para ello se dimensionaron los tiempos en que ocurrieron cada uno de los hechos en un calendario cósmico, dando sus principales características e implicaciones hoy en día, el cual se implementó a modo de una serie de videos, el primero fue un fragmento del Capitulo uno del documental Cosmos Edición 2014, los siguientes manejaban temas específicos como el planteamiento de la teoría del Big Bang y la extinción de los dinosaurios. Al final se realizó una socialización grupal con el fin de la construcción del nuevo conocimiento partiendo de sus dudas, apreciaciones de eventos abordados en los videos para concluir en la elaboración de una línea de tiempo que les permitiera concebir los hechos en un orden cronológico.

En la quita sesión denominada Caracteres evolutivos humanos, los estudiantes observaron las diferencias en su cuerpo con rasgos de sus compañeros, en un primer momento con compañeros de sexo contrario y luego de su mismo género y empezaron a cuestionarse en qué aspectos ha cambiado y las posibles causas de ese cambio; se plantearon las siguientes preguntas: ¿el dimorfismo sexual jugo un papel importante en la evolución humana?, ¿en qué aspectos es diferente el esqueleto humano femenino del masculino?; los alumnos partieron de sus ideas previas para construir un conocimiento mucho más científico y estructurado.

En la sexta sesión, denominada Identificación de cráneos humanos, se pensó en complementar la sesión anterior: Caracteres evolutivos humanos, con el fin de que los estudiantes por medio de tacto y la observación de cráneos se cuestionaran acerca del sexo, si es masculino, femenino o infantil, de tal manera de que el estudiante se viera en la obligación de adquirir nuevos saberes con el fin de genera la motivación para alcanzar el nuevo saber.

En la séptima sesión se realizó la evaluación final en la cual se corroboró el cambio conceptual que tuvo el estudiante con respecto a los temas vistos en clase; durante las 
Bio - grafia. Escritos sobre la Biología y su Enseñanza. ISSN 2027

Edición Extraordinaria. p.p. 1348-1359

Memorias del VIII Encuentro Nacional de Experiencias en Enseñanza de la Biología y la Educación Ambiental. III Congreso Nacional de Investigación en Enseñanza de la Biología.

primeras sesiones los estudiantes definían el conceptos como: "un fósil es encontrar un esqueleto de hace muchos años", "el Big Bang es cuando se extinguieron los dinosaurios", "nosotros venimos de los monos", sin embargo durante el desarrollo de la unidad didáctica hubo una reconfiguración de conceptos: "un fósil es cualquier evidencia de vida que se ha preservado por más de 10.000 años", "Existen fósiles formados por los organismos mismos - partes desmembradas, se llaman: fósiles corpóreos", "Los fósiles que preservan evidencias del comportamiento, tales como las huellas, las perforaciones se llaman: trazas fósiles", "La teoría del Big Bang es diferente a la teoría de la extinción de los dinosaurios".

Al analizar la experiencia pedagógica en la institución con los estudiantes cuyo entorno social era contrastante, dado que la ubicación del colegio se encuentra remitida a un barrio popular en una zona de alto riesgo, se evidencia el poco interés que presentan los mismos por el colegio como mecanismo que les permita acceder al conocimiento y así ampliar su visión de la realidad generando transformaciones de gran impacto en sus vidas, con lo cual el docente titular parece estar de acuerdo limitando su desempeño a dar bases de los temas, según lo observado, no de manera rigurosa y permitir al alumno dejar pasar por alto la exigencia, que posiblemente inmiscuya en su desarrollo en este mundo globalizado donde se rige por una economía del conocimiento y la competitividad entre los individuos que integran la sociedad. Colocando en desventaja a los estudiantes al entrar a competir en el ingreso de una formación educativa superior, recayendo en la misma problemática social de inequidad. Es posible trabajar con este tipo de población, como se constata en el trabajo, desde un enfoque didáctico que posibilite el interés del estudiante por el saber, integrando sus necesidades y los conocimientos que rigen a las ciencias sin que se dé una brecha entre ellos, impulsando el avance en el ámbito educativo y enriqueciendo estos procesos.

Los resultados / productos pueden clasificarse en tres categorías, las cuales pueden potencializar su aplicación:

\section{Relacionados con la generación de conocimiento y/o nuevos desarrollos tecnológicos}

Se busca potencializar el aprendizaje de los alumnos a partir del uso de herramientas tecnológicas sin hacer un mal uso de ellas, por lo cual se plantea la creación de un juego al estilo del programa quien quiere ser millonario, que permita interactuar con los estudiantes y a la ves evaluar la asimilación y apropiación de conocimientos sobre el tema de evolución. 
Bio - grafia. Escritos sobre la Biología y su Enseñanza. ISSN 2027

Edición Extraordinaria. p.p. 1348-1359

Memorias del VIII Encuentro Nacional de Experiencias en Enseñanza de la Biología y la Educación Ambiental. III Congreso Nacional de Investigación en Enseñanza de la Biología.

Siendo posiblemente una gran herramienta de la cual podrían hacer uso los docentes del área de biología e inclusive los mismos alumnos interesados en aprender más sobre el tema de forma divertida.

\section{Conducentes al fortalecimiento de la capacidad científica nacional}

Reconocer el gran valor paleontológico de los restos fósiles encontrados en el territorio nacional, los estudios realizados en el campo de la genética, sistemática y filogenia que permiten vislumbrar las condiciones históricas y los cambios evolutivos acontecidos en Colombia. Contribuyendo en despertar el interés por el tema entre los alumnos y así posiblemente encaminarlos en su formación de nuevos investigadores en un futuro en ramas disciplinares que contemplen el estudio evolutivo.

Incentivando la investigación científica desde las aulas de clase con actividades que comprometan al estudiante a realizar un trabajo riguroso semejante al realizado por un investigador, posibilita en ellos el interés por la ciencia y su gran valor contributivo con entrever nuevos conocimientos que nos acerquen a la verdad.

\section{Dirigidos a la apropiación social del conocimiento}

Como resultado se plantea la concientización por parte de los estudiantes, de los factores ambientales como interventores en los cambios evolutivos presentados por los seres vivientes en cada etapa de las eras geológicas y los ecosistemas, además de ser determinantes para el surgimiento y establecimiento de la vida. Posibilitando una postura crítica frente a la responsabilidad del ser humano en el desequilibrio de la naturaleza y la búsqueda de soluciones viables que permitan contribuir con el cuidado del planeta desde la aprobación e iniciativas llevadas a cabo desde el territorio local.

\section{Impactos esperados a partir del uso de los resultados}

Los impactos esperados fueron un cambio de hábitos que contribuyan con el cuidado del medio ambiente, iniciativas implementadas en el aula de clase que medien en la construcción de nuevos conocimientos científicos y la constitución de proyectos investigativos.

\section{CONCLUSIONES}


Bio - grafia. Escritos sobre la Biología y su Enseñanza. ISSN 2027

Edición Extraordinaria. p.p. 1348-1359

Memorias del VIII Encuentro Nacional de Experiencias en Enseñanza de la Biología y la Educación Ambiental. III Congreso Nacional de Investigación en Enseñanza de la Biología.

- Los estudiantes mostraron un mayor interés por las ciencias en medida que se iba desarrollando la unidad didáctica lo anterior lo atribuimos a que el estudiante propuso de manera activa soluciones, investigó y evaluó sus soluciones y se adecuó frente a las nuevas posibilidades del modelo científico, poniendo a prueba su conocimiento en la resolución de problemas.

- Es importante tener en cuenta las actitudes y fortalezas de los estudiantes con el fin de explorarlas hacia el conocimiento científico.

- La primera reacción de los estudiantes ante el enfoque pedagógico fue la negación y el desentendimiento sin embargo en la medida en que se acercó al estudiante al nuevo concepto desde lo cotidiano, este empezó a indagar, investigar y descubrir.

- Al despertar el interés de los estudiantes, nos permitió desarrollar el componente afectivo el cual los condujo al fortalecimiento y manejo del aprendizaje científico.

\section{REFERENCIAS BIBLIOGRÁFICAS}

Pozo, J.I. (1998). Del conocimiento cotidiano al conocimiento científico: más allá del cambio conceptual. En J.I. Pozo \& M.A. Gómez (Eds.), Aprender y enseñar ciencia: Del conocimiento cotidiano al conocimiento científico (págs. 128-146). Madrid, España: Ediciones Morata.

Pozo, J.I. (1994). El Aprendizaje y La Enseñanza De Hechos y Conceptos. En C. Coll, J.I. Pozo, B. Sarabia \& E. Valls (Eds.), Los contenidos de la Reforma: Enseñanza y aprendizaje de conceptos, procedimientos y actitudes (págs. 19-79). España. Ediciones Santillana.

MEN. Estándares básicos de competencias en Lenguaje, Matemáticas, Ciencias y Ciudadanas. Guía sobre lo que los estudiantes deben saber y saber hacer con lo que aprenden. 2006

Ontoria, Antonio - Molina, Ana - Sánchez, Ángela (1996) Autoconciencia del conflicto cognitivo ante la innovación metodológica en la formación inicial de maestros. Asociación Universitaria de Formación del Profesorado (AUFOP) VIII Congreso de Formación del Profesorado. Revista Electrónica Interuniversitaria de Formación del Profesorado. 
Bio - grafia. Escritos sobre la Biología y su Enseñanza. ISSN 2027

Edición Extraordinaria. p.p. 1348-1359

Memorias del VIII Encuentro Nacional de Experiencias en Enseñanza de la Biología y la Educación Ambiental. III Congreso Nacional de Investigación en Enseñanza de la Biología.

Perales- Palacios, Javier (1992) Desarrollo cognitivo y modelo constructivista en la Enseñanza-Aprendizaje de las ciencias. Revista Interuniversitaria de Formación del Profesorado, No 13. Pp 173-189.

Pozo, Juan Ignacio (1997) Teorías cognitivas del aprendizaje. Editorial Morata. Madrid. Capítulo 8: Enfoques para la enseñanza de la ciencia. Pp 265-308.

Villani, Alberto - Orquiza de Carvalho, Lizete (1995) Conflictos cognitivos, experimentos cualitativos y actividades didácticas. Investigación y experiencias didácticas- Enseñanza de las ciencias. Pp 279-294 